18

\title{
Роль электрон-электронного отталкивания в задаче об эпитаксиальном графене на металле: простые оценки
}

\author{
(C) С.Ю. Давыдов \\ Физико-технический институт им. А.Ф. Иофрфе РАН, \\ Санкт-Петербург, Россия \\ Санкт-Петербургский национальный исследовательский университет \\ информационных технологий, механики и оптики, \\ Санкт-Петербург, Россия \\ E-mail: Sergei_Davydov@mail.ru \\ (Поступила в Редакцию 12 января 2017 г. \\ В окончательной редакции 5 февраля 2017 г.)
}

\begin{abstract}
Для однослойного графена, находящегося на поверхности металлической подложки, в рамках расширенной теории Хартри-Фока рассмотрено влияние интра- и интератомного кулоновского отталкивания электронов $(U$ и $G$ соответственно) на его фазовую диаграмму. Приведено общее решение задачи, на основе которого проанализирован ряд частных случаев, допускающих аналитическое рассмотрение: свободный и эпитаксиальный графен без учета и с учетом энергии перехода электрона между соседними атомами графена. Рассмотрены три области фазовой диаграммы: волны спиновой и зарядовой плотностей (ВСП и ВЗП соответственно) и однородное по спину и заряду состояние полуметалла (ПМ). Основное внимание уделено недопированному графену. Показано, что учет взаимодействия с металлической подложкой расширяет область существования ПМ. Для всех рассмотренных случаев, однако, граница между состояниями ВСП и ВЗП описывается уравнением $U=z G$, где $z=3$ - число ближайших соседей в графене. К уширению области ПМ-состояния приводит и допирование графена, причем эффект не зависит от знака свободных носителей, вносимых в эпитаксиальный графен подложкой. Согласно сделанным оценкам, в буферном слое возможно только ПМ-состояние металлического типа, тогда как в квазисвободном эпитаксиальном графене может быть реализовано ВЗП-состояние. Обсуждается влияние температуры на фазовую диаграмму эпитаксиального графена.
\end{abstract}

DOI: 10.21883/FTT.2017.08.44772.02

\section{1. Введение}

Роль межэлектронного взаимодействия в теории графена всегда вызывала большой интерес $[1,2]$. Из недавних публикаций в этой области отметим работы [3-8], где для описания электронного спектра свободного однослойного графена использовались два варианта модели Хаббарда: стандартный, в рамках которого учитывается только внутриатомная корреляция электронов $U$, и расширенный, где дополнительно включено межатомное взаимодействие электронов $G$. В этих работах обсуждается возможность переходов между стандартным состоянием дираковского полуметалла (ПМ), в котором каждому атому углерода соответствует один электрон с нулевым результирующим спиновым моментом, в состояния с неоднородным спиновым и зарядовым распределением электронов по подрешеткам. Рассматриваются антиферромагнитное распределение электронов, когда числа заполнения $n_{A}$ и $n_{B}$ подрешеток $A$ и $B$ одинаковы, a спиновые моменты противоположно направлены, и состояние с $n_{A} \neq n_{B}$ и нулевыми спиновыми моментами. В первом случае говорят о волне спиновой плотности (ВСП), во втором - о волне зарядовой плотности (ВЗП). В отличие от бесщелевого электронного спектра дираковского ПМ ВСП- и ВЗП-состояния в свободном графене обладают щелью (запрещенной зоной) и являются моттовскими изоляторами.
В настоящей работе обратимся к эпитаксиальному графену (ЭГ) на металлической подложке. Рассмотрим общую задачу о влиянии корреляций $U$ и $G$ на электронную структуру ЭГ. Для этого воспользуемся, во-первых, приближением, впервые введенным в [9] для описания адсорбированных слоев. Сущность этого приближения состоит в том, что мы строим гексагональную решетку ЭГ не из атомов углерода, учитывая затем взаимодействие этой решетки с подложкой, а сразу из адатомов углерода, что схематически показано на рис. 1 (см. подробнее [10,11]). Во-вторых, мы используем

$$
\begin{aligned}
& a-\text { atom interaction } \\
& b \text { - adatom interaction }
\end{aligned}
$$
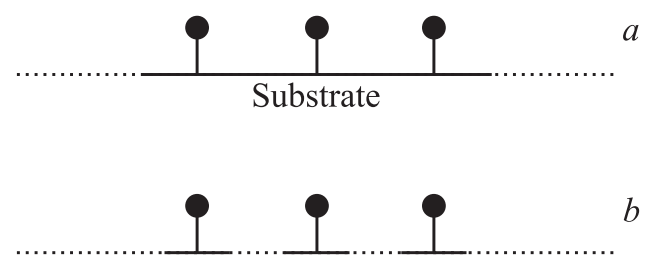

Рис. 1. Схема замены адсорбированной на подложке решетки атомов $(a)$ на решетку адатомов $(b)$. 
некоторые подходы работы [12], в которой рассматривалась задача о неоднородном распределении заряда и намагниченности в адсорбированном на металлической подложке слое и применялась модель с хаббардовской решеткой андерсоновских примесей [13-15]. Мы также рассмотрим ряд частных случаев, для которых в аналитическом виде получим критерии существования областей неоднородных распределений спина и заряда, и обсудим роль температуры.

\section{2. Общее рассмотрение}

Представим гамильтониан системы $H$ в виде

$$
H=H_{\text {sub }}+H_{\mathrm{Gr}}+H_{\text {int }} .
$$

Здесь гамильтониан, отвечающий субстрату, есть

$$
H_{\mathrm{sub}}=\sum_{\mathbf{q}, \sigma} \varepsilon_{\mathrm{sub}}(\mathbf{q}) \hat{n}_{\mathbf{q}, \sigma},
$$

гамильтониан, описывающий графен, равен

$$
\begin{gathered}
H_{\mathrm{Gr}}=H_{\mathrm{Gr}}^{0}+H_{\mathrm{Gr}}^{\mathrm{int}}, \\
H_{\mathrm{Gr}}^{0}=\varepsilon_{0} \sum_{i} \hat{n}_{i}+U \sum_{i} \hat{n}_{i \uparrow} \hat{n}_{i \downarrow} \\
H_{\mathrm{Gr}}^{\mathrm{int}}=-t \sum_{\langle i, j\rangle, \sigma}\left(a_{i \sigma}^{+} a_{j \sigma}+\text { h.c. }\right)+G \sum_{\langle i, j\rangle} \hat{n}_{i} \hat{n}_{j}
\end{gathered}
$$

и гамильтониан взаимодействия находящихся в узлах $i$ изолированных атомов графена с субстратом имеет вид

$$
H_{\text {int }}=\sum_{\mathbf{q}, i, \sigma} V_{i \mathbf{q}}\left(c_{\mathbf{q} \sigma}^{+} a_{\sigma}+\text { h.c. }\right),
$$

где $\hat{n}_{\mathbf{q} \sigma}$ - оператор числа заполнения состояния $|\mathbf{q}, \sigma|$ с волновым вектором q и проекцией спина $\sigma=(\uparrow, \downarrow)$, $c_{\mathbf{q} \sigma}^{+}\left(c_{\mathbf{q} \sigma}\right)-$ соответствующий оператор рождения (уничтожения) электрона подложки, $\hat{n}_{i \sigma}=a_{i \sigma}^{+} a_{i \xi}-$ оператор числа заполнения состояния $i$-го узла решетки графена $|i, \sigma\rangle, a_{i \sigma}^{+}\left(a_{i \sigma}\right)$ - соответствующий оператор рождения (уничтожения) электрона графена, $n_{i}=\sum_{\sigma} \hat{n}_{i \sigma}$, $\varepsilon_{\text {sub }}(\mathbf{q})$ - закон дисперсии электронов подложки, $\varepsilon_{0}-$ энергия состояния $\left|p_{z}\right\rangle$ изолированного атома углерода (точка Дирака), $t$ - энергия перехода электрона между ближайшими соседями (БС) в графене, $V_{i q}$ - матричный элемент взаимодействия, спаривающий состояния $|\mathbf{q}, \sigma\rangle$ и $|i \sigma\rangle$, который в дальнейшем принимается равным константе $V[11-16],\langle i, j\rangle$ обозначает суммирование по БС в графене, h. с. - эрмитово сопряженные слагаемые.

В приближении Хартри-Фока два последних слагаемых выражения (3) можно записать в виде

$$
\begin{array}{r}
U \sum_{i} \hat{n}_{i \uparrow} \hat{n}_{A \downarrow}=U\left(n_{A \uparrow} \hat{n}_{A \downarrow}+n_{A \downarrow} \hat{n}_{A \uparrow}-n_{A \uparrow} n_{A \downarrow}\right. \\
\left.+n_{B \uparrow} \hat{n}_{B \downarrow}+n_{B \downarrow} \hat{n}_{B \uparrow}-n_{B \uparrow} n_{B \downarrow}\right), \\
G \sum_{\langle i, j\rangle} \hat{n}_{i} \hat{n}_{j}=z G\left(n_{A} \hat{n}_{B}+n_{B} \hat{n}_{A}-n_{A} n_{B}\right),
\end{array}
$$

где $z=3$ - число БС в гексагональной решетке, $n_{i \sigma}=\left\langle\hat{n}_{i \sigma}\right\rangle, n_{i}=\left\langle\hat{n}_{i}\right\rangle,\langle\ldots\rangle-$ усреднение по основному состоянию ${ }^{1}$. Вводя энергии

$$
\begin{aligned}
& \varepsilon_{A \uparrow, \downarrow}=\varepsilon_{0}+U n_{A \downarrow, \uparrow}+z G n_{B}, \\
& \varepsilon_{B \uparrow, \downarrow}=\varepsilon_{0}+U n_{B \downarrow, \uparrow}+z G n_{A},
\end{aligned}
$$

запишем гамильтониан $H_{\mathrm{Gr}}$ в приближении ХартриФока:

$$
\begin{aligned}
H_{\mathrm{Gr}}^{\mathrm{HE}}= & -t \sum_{\langle i, j\rangle, \sigma}\left(a_{i \sigma}^{+} a_{j \sigma}+\text { h.c. }\right)+\sum_{\alpha, \sigma} \varepsilon_{\alpha, \sigma} \hat{n}_{\alpha, \sigma} \\
& -U \sum_{\alpha} n_{\alpha \uparrow} n_{\alpha \downarrow}-z G n_{A} n_{B},
\end{aligned}
$$

где $\alpha=A, B-$ индекс подрешетки.

Как уже отмечалось выше, для нахождения функции Грина, соответствующей гамильтониану (1)-(3), воспользуемся адсорбционным приближением [9-12], а также результатами работы [17], где рассматривались эпитаксиальные графеноподобные слои бинарных соединений $^{2}$. Тогда с учетом (7) и (8) получим следующие выражения для диагональных функций Грина подрешеток $A$ и $B$ :

$$
G_{A A(B B)}^{\sigma}(\omega, \mathbf{k})=\frac{\Omega_{B(A)}^{\sigma}+i \Gamma(\omega)}{\left(\Omega_{A}^{\sigma}+i \Gamma(\omega)\right)\left(\Omega_{B}^{\sigma}+i \Gamma(\omega)\right)-t^{2} f^{2}(\mathbf{k})},
$$

где

$$
\begin{aligned}
& f(\mathbf{k})= \\
& =\sqrt{3+2 \cos \left(k_{x} a_{0} \sqrt{3}\right)+4 \cos \left(k_{x} a_{0} \sqrt{3} / 2\right) \cos \left(3 k_{y} a_{0} / 2\right) .}
\end{aligned}
$$

Здесь $\mathbf{k}=\left(k_{x}, k_{y}\right)$ - волновой вектор для движения электрона в плоскости листа графена, $a_{0}$ - расстояния между БС в графене, $\omega$ - энергетическая переменная,

$$
\begin{gathered}
\Omega_{A(B)}^{\sigma}=\omega-\varepsilon_{\sigma}(\omega) \mp \Delta_{\sigma}(\omega), \quad \varepsilon_{\sigma}(\omega)=\left(\tilde{\varepsilon}_{A \sigma}+\tilde{\varepsilon}_{B \sigma}\right) / 2, \\
\Delta_{\sigma}(\omega)=\left(\tilde{\varepsilon}_{A \sigma}-\tilde{\varepsilon}_{B \sigma}\right) / 2, \quad \tilde{\varepsilon}_{A(B) \sigma}=\varepsilon_{A(B) \sigma}+\Lambda(\omega), \\
\Gamma(\omega)=\pi V^{2} \rho_{\text {sub }}(\omega), \quad \Lambda(\omega)=V^{2} P \int_{-\infty}^{\infty} \frac{\rho_{\text {sub }}\left(\omega^{\prime}\right) d \omega^{\prime}}{\omega-\omega^{\prime}},
\end{gathered}
$$

где $\rho_{\mathrm{sub}}(\omega)$ - плотность состояния подложки, и $P-$ символ главного значения. Электронный спектр системы определяется из уравнения $\Omega_{A}^{\sigma} \Omega_{B}^{\sigma}=t^{2} f^{2}$, что дает

$$
\begin{gathered}
E_{ \pm}^{\sigma}(\omega, \mathbf{k})=\varepsilon_{\sigma}(\omega) \pm R_{\sigma}(\omega, \mathbf{k}) \\
R_{\sigma}(\omega, \mathbf{k}) \sqrt{\Delta_{\sigma}^{2}(\omega)+t^{2} f^{2}(\mathbf{k})} .
\end{gathered}
$$

\footnotetext{
${ }^{1}$ Следует отметить, что последний член в (3) отличается от выражения, использованного в [12]. Однако в приближении ХартриФока операторная структура гамильтонианов здесь и в [12] идентична, а различия сводятся лишь к сдвигу энергии $\varepsilon_{0}$.

2 Адсорбционный подход позволяет не учитывать косвенный обмен адатомов углерода через состояния подложки, порождаемый взаимодействием (4).
} 
Отметим, что знак „минус“ соответствует валентной $\pi$-зоне, знак „Плюс ${ }^{“}-\pi^{*}$-зоне проводимости. Плотности состояний в расчете на элементарную ячейку, отвечающие функциям Грина (9), равны

$$
\begin{gathered}
\rho_{A B}^{\sigma}(\omega)=\rho_{A}^{\sigma}(\omega)+\rho_{B}^{\sigma}(\omega), \\
\rho_{A(B)}^{\sigma}(\omega)=-\frac{1}{\pi N} \sum_{\mathbf{k}} \operatorname{Im} G_{A A(B B)}^{\sigma}(\omega, \mathbf{k}),
\end{gathered}
$$

где $\rho_{A(B)}^{\sigma}(\omega)-$ плотность состояний на узле подрешетки $A(B), N=N_{A}=N_{B}$ - число атомов в подрешетках $A$ и $B$, суммирование ведется по первой зоне Бриллюэна. Энергия системы есть

$$
\begin{aligned}
E= & \sum_{\alpha, \sigma} \varepsilon_{\alpha \sigma} \hat{n}_{\alpha \sigma}-U \sum_{\alpha} n_{\alpha \uparrow} n_{\alpha \downarrow}-z G n_{A} n_{B} \\
& +\sum_{\alpha, \sigma} \int_{-\infty}^{\varepsilon_{\mathrm{F}}-\varepsilon_{\alpha \sigma}} \Omega_{\sigma} \rho_{\alpha}^{\sigma}\left(\Omega_{\sigma}\right) d \Omega_{\sigma}+E_{\mathrm{sub}}
\end{aligned}
$$

где $\varepsilon_{\mathrm{F}}-$ уровень Ферми, $\rho_{\alpha}^{\sigma}\left(\Omega_{\sigma}\right)-$ плотность состояний для $\alpha$-подрешетки (на атом), $E_{\text {sub }}$ - вклад субстрата в энергию системы.

\section{3. Свободный графен}

Рассмотрим частные случаи, полезные для дальнейшего анализа. Начнем со свободного графена, описываемого гамильтонианом (8), в пределе сильной корреляции $U, G \gg t$. Полагая в нулевом приближении $t=0$, получим для энергии $E_{\mathrm{Gr}}^{\mathrm{HE}}\left(n_{A \uparrow}, n_{A \downarrow} \mid n_{B \uparrow}, n_{b \downarrow}\right) \equiv\left\langle H_{\mathrm{Gr}}^{\mathrm{HE}}\right\rangle$ выражение

$$
\begin{aligned}
E_{\mathrm{Gr}}^{\mathrm{HE}}\left(n_{A \uparrow},\right. & \left.n_{A \downarrow} \mid n_{B \uparrow}, n_{B \downarrow}\right)=\varepsilon_{0}\left(n_{A}+n_{B}\right) \\
& +U\left(n_{A \uparrow} n_{A \downarrow}+n_{B \uparrow} n_{B \downarrow}\right)+z G n_{A} n_{B} .
\end{aligned}
$$

Если на одну элементарную ячейку приходятся два электрона, для случая антиферромагнитного спинового упорядочения, или при наличии ВСП, имеем $E_{\mathrm{Gr}}^{\mathrm{HE}}(1,0 \mid 0,1) \approx 2 \varepsilon_{0}+z G$. В том случае, когда на узлах подрешетки $A$ находятся два электрона, а узлы подрешетки $B$ пусты, имеет место ВЗП, характеризуемая энергией $E_{\mathrm{Gr}}^{\mathrm{HE}}(1,1 \mid 0,0) \approx 2 \varepsilon_{0}+U$. Таким образом, при $z G<U$ имеем ВСП-состояние, при $z G>U-$ ВЗП-состояние. Это хорошо известный результат, не связанный непосредственно ни с приближением Хартри-Фока, ни с геометрией и размерностью решетки (при одинаковых значениях $z$ ) [18-22]. Более того, численные расчеты [18-22] показывают, что граница, разделяющая ВСП- и ВЗП-состояния, с хорошей точностью описывается уравнением $z G=U$. Далее на примере простых моделей, допускающих аналитическое рассмотрение, продемонстрируем справедливость этого вывода в том числе и для ЭГ.
Для дальнейшего анализа введем, как и в [12] (см. также [11]), следующие параметры:

$2 a=n_{A \uparrow}+n_{A \downarrow}+n_{B \uparrow}+n_{B \downarrow}, 2 b=n_{A \uparrow}-n_{A \downarrow}+n_{B \uparrow}-n_{B \downarrow}$,
$2 c=n_{A \uparrow}+n_{A \downarrow}-n_{B \uparrow}-n_{B \downarrow}, 2 d=n_{A \uparrow}-n_{A \downarrow}-n_{B \uparrow}+n_{B \downarrow}$.

Величины $2 b, 2 c$ и $2 d$ являются параметрами порядка, описывающими соответственно намагниченность, ВЗПи ВСП-состояния двухатомной элементарной ячейки ЭГ, содержащей $2 a$ электронов. Тогда

$$
\begin{gathered}
n_{A \uparrow, \downarrow}=(a \pm b+c \pm d) / 2, \quad n_{B \uparrow, \downarrow}=(a \pm b-c \mp d) / 2, \\
\varepsilon_{A \uparrow, \downarrow}=\varepsilon_{0}+U(a \mp b+c \mp d) / 2+z G(a-c), \\
\varepsilon_{B \uparrow, \downarrow}=\varepsilon_{0}+U(a \mp b+c \pm d) / 2+z G(a+c) .
\end{gathered}
$$

Положим в дальнейшем $\varepsilon^{*} \equiv \varepsilon_{0}+a(U / 2+z G)=0$. В новых обозначениях выражение (15) приобретает вид

$$
E_{\mathrm{Gr}}^{\mathrm{HF}}=E_{0}-\left(b^{2}+d^{2}\right) U / 2+c^{2}(U / 2-z G),
$$

где $E_{0}=-(U / 2)+z G$.

Будем по-прежнему рассматривать свободный графен, но учтем теперь межатомный обмен электронами, т.е. $t$-взаимодействие. Как показано в [17], в низкоэнергетическом приближении плотность состояний свободного графена на элементарную ячейку равна

$$
\rho_{A B}^{\sigma}\left(\Omega_{\sigma}\right)= \begin{cases}\frac{2\left|\Omega_{\sigma}\right|}{\xi^{2}}, & \sqrt{\xi^{2}+\Delta_{\sigma}^{2}} \geq\left|\Omega_{\sigma}\right| \geq\left|\Delta_{\sigma}\right|, \\ 0, & \left|\Omega_{\sigma}\right|<\left|\Delta_{\sigma}\right|,\left|\Omega_{\sigma}\right|>\sqrt{\xi^{2}+\Delta_{\sigma}^{2}} .\end{cases}
$$

Здесь $\Omega_{\sigma}=\omega-\varepsilon_{\sigma}, \varepsilon_{\sigma}=\left(\varepsilon_{A \sigma}+\varepsilon_{B \sigma}\right) / 2, \Delta_{\sigma}=\left(\varepsilon_{A \sigma}-\varepsilon_{B \sigma}\right) / 2$, $\xi=\sqrt{2 \pi \sqrt{3}} t$.

Легко показать, что при этом $\varepsilon^{*}=\varepsilon_{\mathrm{F}}$, и получаем $a=1$. Это так называемый симметричный случай $[11,23]$, когда $n_{A, B}=1 \pm c[11,12]$. Положим $b=0$, откуда $m_{A}=-m_{B}=d$, где $m_{A, B}=n_{A \uparrow, B \uparrow}-n_{A \downarrow, B \downarrow}$, что соответствует антиферромагнитному состоянию. Тогда возможны следующие ситуации: $\varepsilon_{A \uparrow}=\varepsilon_{B \downarrow}=-d U / 2$, $\varepsilon_{A \downarrow}=\varepsilon_{B \uparrow}=d U / 2 \quad$ при $\quad c=0, \quad d \neq 0 \quad$ (ВСП); $\varepsilon_{A \uparrow}=\varepsilon_{A \downarrow}=c(U / 2-z G), \quad \varepsilon_{B \uparrow}=\varepsilon_{B \downarrow}=-c(U / 2-z G)$ при $c \neq 0, d=0$ (ВЗП); $\varepsilon_{\alpha \sigma}=0 \quad$ при $\quad c=d=0$ (ПМ). Поскольку $\varepsilon_{\sigma}=0$ и $\Delta_{\uparrow, \downarrow}=U(c \mp d) / 2-c z G$, соответствующие законы дисперсии имеют вид $E_{ \pm}^{\sigma}(\mathbf{k})= \pm \sqrt{(d U / 2)^{2}+t^{2} f^{2}(\mathbf{k})} \quad\left(\mathrm{BC \Pi ),} \quad E_{ \pm}^{\sigma}(\mathbf{k})=\right.$ $= \pm \sqrt{c^{2}(U / 2-z G)^{2}+t^{2} f^{2}(\mathbf{k})}\left(\right.$ В3П), $E_{ \pm}^{\sigma}(\mathbf{k})= \pm t|f(\mathbf{k})|$ (ПМ). Таким образом, бесщелевое дираковское ПМсостояние качественно отличается от имеющих щели состояний ВСП и ВЗП, представляющих собой моттовский изолятор.

Энергия $\bar{E}(a=1, b=0, c, d) \equiv \bar{E}(c, d)$ рассматриваемой системы определяется выражением

$$
\bar{E}(c, d)=-\frac{2}{3 \xi^{2}} \sum_{\sigma}\left(\left(\xi^{2}+\Delta_{\sigma}^{2}\right)^{3 / 2}-\left|\Delta_{\sigma}\right|^{3}\right)+\bar{E}_{\mathrm{Gr}}^{\mathrm{HF}},
$$

где $\bar{E}_{\mathrm{Gr}}^{\mathrm{HF}}$ дается формулой (19) при $b=0$. Анализ показал (см. Приложение, пункт 1), что граница между состоя- 
ниями ВСП и ВЗП описывается условием $z G=U$. При этом совпадают и соответствующие законы дисперсии.

\section{4. Эпитаксиальный графен: частные случаи}

4.1. Недопированный эпитаксиальный гра$\phi$ ен при $t=0$. Учтем взаимодействие атомов графена с подложкой (4), но пренебрежем их взаимодействием между собой, положив $t=0$. Этот случай является нулевым приближением режима сильной связи графена с субстратом [24]. В качестве подложки рассмотрим металл, считая $\rho_{\mathrm{sub}}(\omega)=\rho_{m}=$ const, так что в приближении бесконечно широкой зоны проводимости металла получаем полуширину квазиуровня адатома углерода $\Gamma(\omega)=\Gamma_{m}=$ const, а гибридизационный сдвиг его квазиуровня $\Lambda(\omega)=0[11,23]$. Тогда приходим к системе четырех уравнений вида

$$
\Gamma_{m} \cot \left(\pi n_{A \sigma(B \sigma)}\right)=\varepsilon_{A \sigma(B \sigma)}-\varepsilon_{\mathrm{F}},
$$

где энергии $\varepsilon_{A \sigma(B \sigma)}$ определяются выражениями (7). Полагая $\varepsilon^{*}=\varepsilon_{\mathrm{F}}$, т. е. $a=1$ и $b=0$, вместо (22) получим систему двух уравнений

$$
\begin{gathered}
\Gamma_{m} \tan [(\pi / 2)(c+d)]=c z G-(c-d) U / 2, \\
\Gamma_{m} \tan [(\pi / 2)(c-d)]=c z G-(c+d) U / 2 .
\end{gathered}
$$

Отсюда следует, что решение $c=0, d \neq 0$, реализуется при выполнении неравенства $U>\pi \Gamma_{m}$, решение $c \neq 0$, $d=0-$ при $2 z G-U>\pi \Gamma_{m}$, решение $c=d \neq 0$ при $z G=U>\pi \Gamma_{m}$.

Для определения области устойчивости найденных решений требуется проанализировать энергию системы $E^{\prime}$. Легко показать, что плотность состояний рассматриваемой системы дается выражением

$$
\rho_{A B}^{\sigma}(\omega)=\frac{\Gamma_{m}}{\pi} \sum_{\alpha} \frac{1}{\left(\omega-\varepsilon_{\alpha \sigma}\right)^{2}+\Gamma_{m}^{2}},
$$

а энергия системы $E^{\prime}(a=1, b=0, c, d) \equiv E^{\prime}(c, d)$ может быть представлена в виде

$$
E^{\prime}(c, d)=\frac{\Gamma_{m}}{2 \pi} \sum_{\alpha, \sigma} \ln \frac{\varepsilon_{\alpha \sigma}^{2}+\Gamma_{m}^{2}}{W^{2}}+\bar{E}_{\mathrm{Gr}}^{\mathrm{HF}}+E_{m},
$$

где значения $\varepsilon_{\alpha \sigma}$ и $n_{\varepsilon \sigma}$ определяются формулами (7) и (15) соответственно, $W$ - энергия обрезания, вводимая в соответствии с приближением бесконечно широкой зоны $[11,12,15,23], E_{m}-$ энергия металлической подложки, которую мы в дальнейшем опускаем, считая ее невозмущенной наличием графена (последнее обстоятельство связано с заданием плотности состояний подложки в виде не зависящей от энергии константы: см. задачу 18.3 в [23]). Анализ полученных результатов (см. Приложение, пункт 2) приводит к фазовой диаграмме,



Рис. 2. Фазовая диаграмма ЭГ для случая $a=1$, $b=0$ (два электрона на элементарную ячейку с нулевой суммарной намагниченностью) при $t=0 . g=G / \pi \Gamma_{m}$ и $u=U / \pi \Gamma_{m}-$ приведенное межатомное и внутриатомное отталкивание электронов, SDW - BCП-состояние $(c=0, d \neq 0)$, CDW - В3П-состояние $(c \neq 0, d=0), \mathrm{SM}-$ ПМ-состояние $(c=0, d=0)$. Прямая 1 отвечает уравнению $u=z g$, прямая $2-$ уравнению $u=1$, прямая $3-u=2 z g-1$.

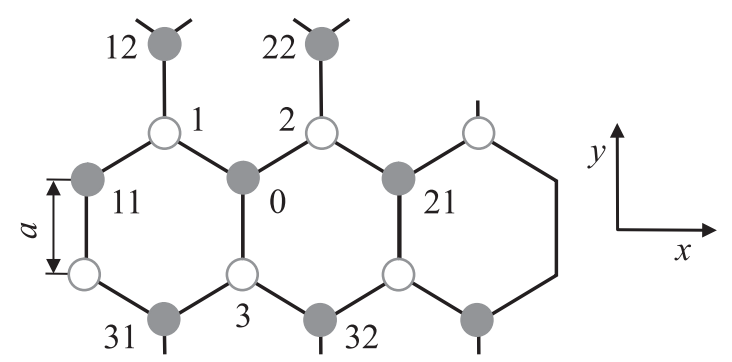

Рис. 3. Решетка адатомов графена с выделенным кластером $0-1-2-3-31-32$. Темными кружками обозначены адатомы подрешетки $A$, светлыми - подрешетки $B$.

изображенной на рис. 2. Отметим, что граница между состояниями ВСП и ВЗП вновь описывается условием $z G=U$.

4.2. Недопированный эпитаксиальный графен при $t \neq 0$. Учтем теперь прямой обмен между атомами ЭГ, для чего рассмотрим кластер из шести адатомов $0-1-2-3-31-32$, изображенных на рис. 3 , заимствованном из работы [17]. Поскольку мы имеем дело с кластером, а не с бесконечной структурой, следует говорить не о ВЗП и ВСП, а о неоднородных зарядовой и спиновой плотностях (НЗП и НСП соответственно).

Результаты рассмотрения приведены в Приложении (пункт 3). Полагая, как и ранее, $a=1, b=0$, т.е. 
$\varepsilon_{\mathrm{F}}=\varepsilon^{*}=0$, вместо (П10) получим

$$
\begin{aligned}
& \pi(c-d)=2 C_{\downarrow} \arctan \left(t_{\downarrow} / \Gamma_{m}\right), \\
& \pi(c+d)=2 C_{\uparrow} \arctan \left(t_{\uparrow} / \Gamma_{m}\right),
\end{aligned}
$$

где $\quad \varepsilon_{ \pm \sigma}= \pm t_{\sigma}, \quad t_{\sigma}= \pm \sqrt{\Delta_{\sigma}^{2}+z t^{2}}, \quad C_{\sigma}=\left|\Delta_{\sigma}\right| / t_{\sigma}$, $\Delta_{\uparrow, \downarrow}=U(c \mp d) / 2-c z G$.

Решение $c \neq 0, d=0$ (НЗП) имеет место при выполнении неравенства $2 z G-U>\pi \Gamma_{m} \eta$, где $\eta=\left(t \sqrt{z} / \Gamma_{m}\right)\left(\arctan \left(t \sqrt{z} / \Gamma_{m}\right)\right)^{-1}>1$. Решение $c=0$, $d \neq 0$ (НСП) возникает при условии $U>\pi \Gamma_{m} \eta$. Граница между НЗП- и НСП-состояниями, соответствующая условию $c=d \neq 0$, отвечает неравенству $U=$ $=z G>\pi \Gamma_{m} \eta_{\max }$, где $\eta_{\max }=\left(t_{\max } / \Gamma_{m}\right)\left(\arctan \left(t_{\max } / \Gamma_{m}\right)\right)^{-1}$ и $t_{\max }=\sqrt{(z G)^{2}+z t^{2}}$. Поскольку множитель $\eta>1$, при включении обменного $t$-взаимодействия область однородного ПМ-состояния расширяется ${ }^{3}$ (см. неравенства после выражений (23)). Соответствующая фазовая диаграмма приведена на рис. 4: в случае $t=2.8 \mathrm{eV}[22]$ и $\Gamma_{m}=10 \mathrm{eV}$ (буферный слой, см. далее) $\eta_{1}=1.07$, тогда как при $\Gamma_{m}=1 \mathrm{eV}$ (квазисвободный графен, см. далее) имеем $\eta_{2}=3.55$.

Полученные в этом подразделе результаты допускают еще одну трактовку. Пусть $\Gamma_{m} \rightarrow 0$, что означает переход к свободному графену с учетом $t$-взаимодействия, но не в зонном варианте (как в пункте 3 ), а в кластерной модели. Тогда для существования НСП необходимо выполнение неравенства $U>2 \sqrt{z} t \approx 3.5 t$, для существования НЗП - неравенства $2 z G-U>2 \sqrt{z} t$. Первую оценку интересно сравнить с суммированными в [3] результатами расчетов различных авторов. Так, например, область существования НСП-состояния в приближении ХартриФока дается неравенством $U>2.2 t$, а в квантовом методе Монте-Карло - неравенством $U>(4.5 \pm 0.5) t$. Таким образом, наша оценка $U>3.5 t$, полученная в рамках кластерной модели, вполне приемлема. Интересно отметить, что значение $U=3.5 t$ является критерием, разделяющим бесщелевую квантовую спиновую жидкость и квантовую спиновую жидкость, обладающую щелью [3,25]. Справедливости ради следует упомянуть и работы [5,26-28], где оценки соотношения между $U$ и $t$ отличаются от приведенных выше.

4.3. Допированный эпитаксиальный графен при $t=0$. Учтем теперь возможность допирования ЭГ подложкой, для чего положим $\varepsilon^{*}-\varepsilon_{\mathrm{F}}=\delta$ и $a=1+v,|v<1|$. Если $v>0$, то электроны переходят из подложки на лист графена, при $v<0$ имеет место обратный процесс. Считая, как и ранее, $b=0$, вместо

\footnotetext{
${ }^{3}$ Мы по-прежнему называем это однородное по спину и заряду состояние полуметаллом. Однако ПМ-состояние ЭГ уже не является дираковским ПМ, как в свободном графене: во-первых, плотность состояний в точке Дирака не обращается в нуль и, во-вторых, в области малых энергий плотность состояний зависит от энергии нелинейно [24]. Аналогичным образом, ВСП- и ВЗП-состояния ЭГ не являются моттовскими изоляторами, так как энергетическая щель отсутствует.
}

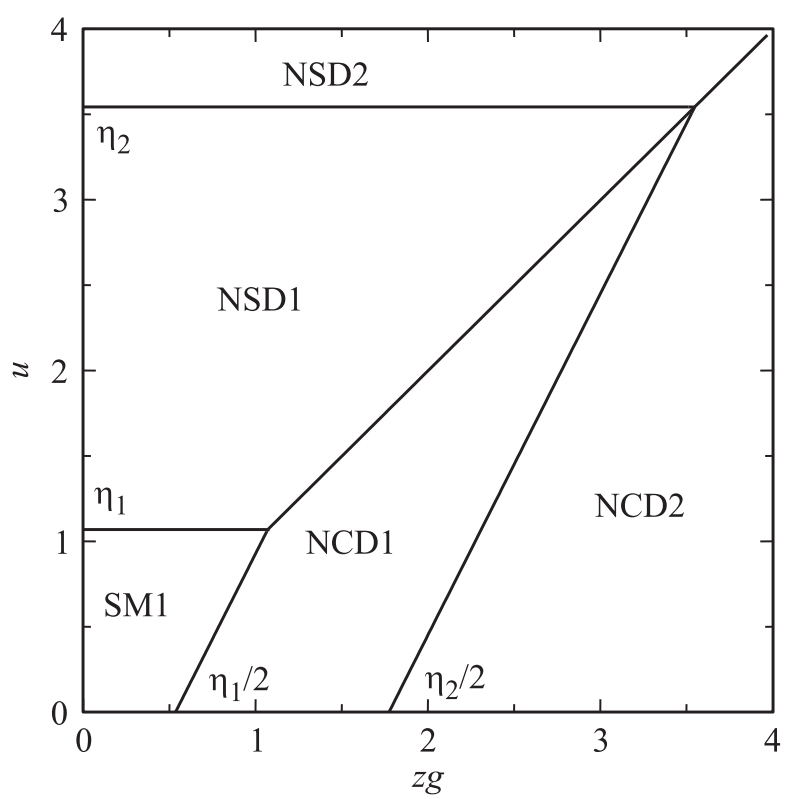

Рис. 4. Фазовая диаграмма для кластера из шести адатомов углерода (рис. 3) при $a=1, b=0$ (два электрона на два адатома в узлах 0 и 3 с нулевой суммарной намагниченностью), $t=2.8 \mathrm{eV}, \Gamma_{m 1}=10 \mathrm{eV}$ (индекс 1) и $\Gamma_{m 2}=1 \mathrm{eV}$ (индекс 2). Множитель $\eta_{i}=\left(t \sqrt{z} / \Gamma_{m i}\right)\left(\operatorname{atrctan}\left(t \sqrt{z} / \Gamma_{m i}\right)\right)^{-1}$, где $i=1$ и 2, определяет расширение области ПМ (SM) и соответствующее сужение областей НСП (NSD) и НЗП (NSD): $\eta_{1}=1.07, \eta_{2}=3.55$. Первому случаю соответствуют области NSD, NCD1, SM1, второму - NSD2, NCD2; область SM2, находящаяся между прямыми, исходящими из точек $u=\eta_{2}$ и $z g=\eta_{2} / 2$, не обозначена, чтобы не перегружать рисунок. Границы областей описываются уравнениями $u=z g, u=\eta$ и $u=2 z g-\eta$ (см. примечания к рис. 2).

системы уравнений (22) имеем

$$
\begin{aligned}
& -\Gamma_{m} \tan [\pi(v+c+d) / 2]=\delta+U(c-d) / 2-c z G, \\
& -\Gamma_{m} \tan [\pi(v+c-d) / 2]=\delta+U(c+d) / 2-c z G .
\end{aligned}
$$

Полагая $|v| \ll 1$, получим $v=-2 \delta \cos ^{2}(\pi d / 2) / \pi \Gamma_{m}$ для ВСП-состояния $(c=0, d \neq 0), v=-2 \delta \cos ^{2}(\pi c / 2) / \pi \Gamma_{m}$ для ВЗП-состояния $(c \neq 0, d=0)$ и $v=-2 \delta / \pi \Gamma_{m}$ для ПМ-состояния $(c=d=0)$.

Легко сообразить, что в случае допированного ЭГ энергия системы дается формулой (25) с заменой $\varepsilon_{\alpha \sigma}^{2}$ на $\left(\varepsilon_{\alpha \sigma}-\varepsilon_{\mathrm{F}}\right)^{2}$. Анализ энергетических характеристик, представленный в пункте 4 Приложения, показывает, что граница между состояниями ВСП и ВЗП по-прежнему описывается условием $z G=U$. Как продемонстрировано там же, значения энергий ВСП, ВЗП и ПМ от знака параметра $\delta$ не зависят. Таким образом, имеется зависимость энергии системы от уровня легирования, но не от знака носителей. Следует отметить также, что в большинстве работ по данной тематике рассматривается недопированный случай. Как, однако, показано в $[27,28]$, допирование может приводить к новым фазовым состояниям двумерной гексагональной структуры. 


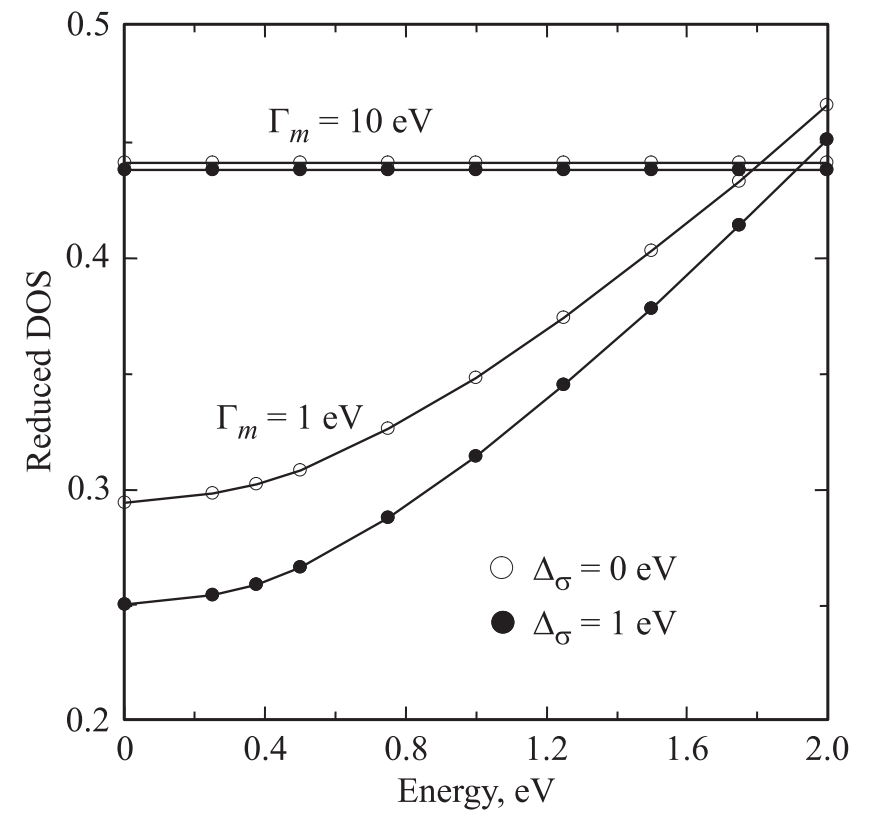

Рис. 5. Приведенная плотность состояний (DOS) $F_{\sigma}(\omega) \equiv \rho_{A B}^{\sigma}(\omega) \xi \quad$ в $\quad$ зависимости $\quad$ от $\quad$ энергии $\quad \omega$ для квазисвободного ЭГ $\left(\Gamma_{m}=1 \mathrm{eV}\right)$ и буферного слоя $\left(\Gamma_{m}=10 \mathrm{eV}\right)$ в отсутствие (светлые кружки) и при наличиии (темные кружки) щели $\Delta_{\sigma}$ в электронном спектре при $a=1$ и $\xi=10 \mathrm{eV}$. Ввиду четности функции $F_{\sigma}(\omega)$ изображена только ее правая часть.

\section{5. Численные оценки и обсуждение полученных результатов}

Перейдем к количественным оценкам параметров задачи и начнем с определения величины $\Gamma_{m}=\pi V^{2} \rho_{\text {sub. }}$. Если предположить, что $p_{z}$-состояния атомов углерода графена связаны с $d$-состояниями переходного металла, то $V=V_{p d \sigma}=\eta_{p d \sigma}\left(\hbar^{2} r_{d}^{3 / 2} / m_{0} l^{7 / 2}\right)$, где $\hbar-$ приведенная постоянная Планка, $m_{0}$ - масса свободного электрона, $r_{d}$ - радиус $d$-состояния, $l$ - длина адсорбционной связи, $\eta_{p d \sigma}=2.95$ [29] (об оценке матричных элементов $V$ см. подробнее [16]). Положим $l_{\min }=r_{\mathrm{C}}+r_{m}$, где $r_{\mathrm{C}}=0.77 \AA-$ атомный радиус углерода, $r_{m}-$ атомный радиус металла. Поскольку для переходных металлов $r_{d} \sim 0.7-1.4 \AA$ [30] и $r_{m} \sim 1.3-1.6 \AA$ [29], получаем $V_{\max } \sim 1-2 \mathrm{eV}$. Далее в соответствии с моделью Фриделя (см., например, [31]) положим для переходных металлов $\rho_{\text {sub }}=N_{d} / W_{d}$, где $W_{d}$ - ширина зоны проводимости, $N_{d}=10$. Так как для $d$-металлов $W_{d} \sim 3-11 \mathrm{eV}$ [31], получаем $\rho_{\text {sub }} \sim 3-1 \mathrm{eV}^{-1}$. Тогда максимальное значение $\Gamma_{m} \sim 10 \mathrm{eV}$. Эта оценка соответствует буферному углеродному слою, сильно связанному с подложкой. Для квазисвободного графена, полагая $l \sim 3 \AA$, имеем $V_{\max } \sim 0.3-0.8 \mathrm{eV}$ и $\Gamma_{m} \sim 1-2 \mathrm{eV}$. Принимая $t=2.8 \mathrm{eV}$ [22], получим $\xi=9.2 \mathrm{eV}$.

Общее выражение для плотности состояний $\rho_{A B}^{\sigma}(\omega)$ определяется формулой (П14) (см. Приложение, пункт 5). На рис. 5 представлены зависимости при- веденной плотности состояний $F_{\sigma}(\omega) \equiv \rho_{A B}^{\sigma}(\omega) \xi$ для $\xi=10 \mathrm{eV}, \Gamma_{m}=1$ и $10 \mathrm{eV}$ и $\Delta_{\sigma}=0$ и $1 \mathrm{eV}$. Поскольку при $a=1$ плотность состояний является четной функцией энергии, на рис. 5 изображена только отвечающая положительным энергиям часть плотности состояний. Ясно видно, что уже в случае квазисвободного ЭГ на металле $\left(\Gamma_{m}=1 \mathrm{eV}\right)$ щель в плотности состояний отсутствует, в чем и состоит главный эффект, вносимый металлическим субстратом (то же относится и к графеноподобным слоям на металлах [17]). При удалении от точки Дирака зависимость $F_{\sigma}$ от $\omega$ приближается к линейной. В случае буферного слоя (при $\Gamma_{m}=10 \mathrm{eV}$ ) все характерные черты графена вообще исчезают: плотность состояний ЭГ становится практически постоянной, характерной для металлической подложки (подробнее см. работы $[17,24]$, где обсуждаются характерные особенности плотности состояний ЭГ).

Перейдем теперь к оценкам кулоновских параметров. В работе [22] для свободного однослойного графена получены следующие эффективные (с учетом экранировки собственными электронами) значения кулоновских параметров: $U=9.3 \mathrm{eV}$ и $G=5.5 \mathrm{eV}$. В отсутствие экранировки эти параметры равны соответственно $U_{0}=17.0 \mathrm{eV}$ и $G_{0}=8.5 \mathrm{eV}[22]$. Экранировка, таким образом, весьма существенно понижает значения $U_{0}$ и $G_{0}{ }^{4}$. Будем считать приведенные выше величины $U$ и $G$ максимальными значениями кулоновских параметров, отдавая себе отчет в том, что электроны металлической подложки усиливают экранировку (некоторые аспекты этой малоизученной проблемы рассматриваются в работах $[4,7,32,33]$. Таким образом, для буферного слоя имеем $U \sim \xi \sim \Gamma_{m}>G$, а для квазисвободного графена получаем $U \sim \xi>G>\Gamma_{m}$.

С учетом найденных параметров рассмотрим фазовую диаграмму кластера из шести углеродных адатомов, представленную на рис. 4. В случае буферного слоя имеем $u \approx 0.3, z g \approx 0.5$ и $\eta \approx 1.1$; для квазисвободного графена $u \approx 3.0, z g \approx 5.3$ и $\eta \approx 3.5$. Отсюда следует, что условие возникновения ВСП-состояния $u>\eta$ заведомо не выполняется для углеродного буферного слоя. Для квазисвободного ЭГ это неравенство также не выполняется, но различие между значениями $u$ и $\eta$ сравнительно мало, так что фазовая точка системы хоть и находится в области ПМ, но вблизи границы с состоянием ВСП. ВЗП-состояние, определяемое условием $2 z g-u>\eta$, в буферном слое не реализуется, тогда как в квазисвободном ЭГ вполне осуществимо. Следовательно, согласно сделанным оценкам, в буферном слое возможно только спиново- и зарядово-однородное ПМ-состояние, тогда как в квазисвободном ЭГ неоднородное ВЗП-состояние может возникнуть.

В заключение этого раздела, обсудим роль температуры. Для расчета чисел заполнения $n_{\alpha \sigma}$ (как и ранее,

\footnotetext{
${ }^{4}$ Отметим, однако, что учет экранировки сравнительно слабо влияет на соотношение между кулоновскими параметрами: $G / U \sim 0.6$ и $G_{0} / U_{0} \sim 0.5$.
} 
$\alpha=A, B-$ индекс подрешетки) нужно умножить плотность состояний $\rho_{\alpha}^{\sigma}(\omega)$ (см. П14) на функцию распределения Ферми-Дирака $f_{\mathrm{FD}}(\omega)$ и взять от этого произведения несобственный интеграл по энергии $\omega$. Поскольку ЭГ на металле является металлической системой, можно воспользоваться результатами работы [34] и по аналогии с приведенной там формулой (3) записать числа заполнения для адатома углерода в виде

$$
\begin{aligned}
\Delta n_{\alpha \sigma}(T) & =n_{\alpha \sigma}(T)-n_{\alpha \sigma}(0) \\
& \approx \frac{\pi}{3} T^{2} \frac{\varepsilon_{\alpha \sigma} \Gamma_{m}}{\left(\varepsilon_{\alpha \sigma}^{2}+\Gamma_{m}^{2}\right)^{2}},
\end{aligned}
$$

где $T-$ температура в энергетических единицах, $\mu(0)=\varepsilon_{\mathrm{F}}=0$ - химический потенциал при нулевой температуре. В случае $\left|\varepsilon_{\alpha \sigma}\right| \ll \Gamma_{m}$ получаем $\Delta n_{\alpha \sigma}(T) \sim\left(T / \Gamma_{m}\right)^{2}\left(\varepsilon_{\alpha \sigma} / \Gamma_{m}\right) ;$ при обратном неравенстве $\left|\varepsilon_{\alpha \sigma}\right| \gg \Gamma_{m}$ имеем $\Delta n_{\alpha \sigma}(T) \sim\left(T / \varepsilon_{\alpha \sigma}\right)^{2}\left(\Gamma_{m} / \varepsilon_{\alpha \sigma}\right)$; если же $\left|\varepsilon_{\alpha \sigma}\right| \sim \Gamma_{m}$, то $\Delta n_{\alpha \sigma}(T) \sim \operatorname{sgn}\left(\varepsilon_{\alpha \sigma}\right)\left(T / \Gamma_{m}\right)^{2}$. Так как $\Gamma_{m} \sim 1-10 \mathrm{eV},\left|\Delta n_{\alpha \sigma}(T)\right| \ll 1$. В настоящей работе мы в основном рассматриваем ситуацию, когда $a=n_{A}+n_{B}=2$. Если $n_{A} \sim n_{B} \sim 1$, то температурные поправки к числам заполнения малы. Этого, однако, нельзя сказать заранее о величинах параметров порядка $c=\left(n_{A}-n_{B}\right) / 2$ при $\left|n_{A}-n_{B}\right| \ll 1$ и $\left|m_{A}-m_{B}\right| \ll 1$. Таким образом, учет конечной температуры влияет лишь на сравнительно узкие полосы ВСП- и ВЗП-областей, примыкающие к границам с ПМ-областью (рис. 4).

\section{6. Заключение}

В настоящей работе мы рассмотрели условия существования трех областей фазовой диаграммы однослойного ЭГ, находящегося на металлической подложке: волны спиновой плотности, волны зарядовой плотности и однородное состояние (ПМ). ВСП- и ВЗП-состояния имеют место исключительно благодаря интра- и интератомному кулоновским взаимодействиям электронов графена, однородное по спину и заряду ПМ-состояние существенно отличается от состояния дираковского полуметалла. Мы показали, что учет прямого обмена $t$ между атомами углерода графена и их взаимодействия с металлом, характеризуемого параметром $\Gamma_{m}$, ведет к сужению областей существования ВСП- и ВЗП-состояний. Установлено, что в буферном слое реализуется только спиново- и зарядово-однородное состояние металлического типа, тогда как в квазисвободном ЭГ может присутствовать ВЗП.

В заключение добавим следующее: то обстоятельство, что ряд результатов других авторов, достигнутых путем численных расчетов в рамках более строгой теории, получен нами простым образом и в аналитической форме, представляется нам достоинством модели, использованной в настоящей работе.

\section{Приложение}

1. Для свободного графена получаем

$\bar{E}(0, d)=E_{0}-\frac{d^{2} U}{2}-\frac{4}{e \xi^{2}}\left[\left(\xi^{2}+(d U / 2)^{2}\right)^{3 / 2}-(d U / 2)^{3}\right]$

при $c=0, d \neq 0$ (ВСП),

$$
\begin{aligned}
\bar{E}(c, 0) & =E_{0}+\frac{c^{2}(U-2 z G)}{2} \\
& -\frac{4}{3 \xi^{2}}\left[\left(\xi^{2}+c^{2}(U / 2-z G)^{2}\right)^{3 / 2}-c^{2}|U / 2-z G|^{3}\right]
\end{aligned}
$$

при $c \neq 0, d=0$ (ВЗП),

$$
\bar{E}(0,0)=E_{0}-\frac{4 \xi}{3}
$$

при $c=d=0 \quad$ (дираковский ПМ). Полагая $\bar{E}(0, d)=\bar{E}(c, 0) \quad$ при $c=d, \quad$ находим условие $U=z G$. Уравнения (П1) и (П2) при $d=0$ и $c=0$ соответственно переходят в уравнение (П3).

2. Для недопированного ЭГ при $t=0$ получаем

$$
E^{\prime}(0, d)=E_{0}-\frac{d^{2} U}{2}+\frac{2 \Gamma_{m}}{\pi} \ln \frac{(d U / 2)^{2}+\Gamma_{m}^{2}}{W^{2}}
$$

при $c=0, d \neq 0(\mathrm{BCП),}$

$$
\begin{aligned}
E^{\prime}(c, 0)= & E_{0}+\frac{c^{2}(U-2 z G)}{2} \\
& +\frac{2 \Gamma_{m}}{\pi} \ln \frac{c^{2}(U / 2-z G)^{2}+\Gamma_{m}^{2}}{W^{2}}
\end{aligned}
$$

при $c \neq 0, d=0$ (ВЗП),

$$
E^{\prime}(0,0)=E_{0}+\frac{2 \Gamma_{m}}{\pi} \ln \frac{\Gamma_{m}^{2}}{W^{2}}
$$

при $c=d=0$ (ПМ). Полагая $\bar{E}(0, d)=\bar{E}(c, 0)$ при $c=d$, находим условие $U=z G$. Уравнения (П4) и (П5) при $d=0$ и $c=0$ соответственно переходят в уравнение (П6).

3. Введем функции Грина невзаимодействующих адатомов и подшеток $A$ и $B$

$$
g_{A(B)}^{\sigma}(\omega)=\left(\omega-\varepsilon_{A(B) \sigma}+i \Gamma_{m}\right)^{-1} .
$$

Рассматривая кластер, изображенный на рис. 3, включая $t$-взаимодействие между адатомами и воспользовавшись уравнением Дайсона, найдем функции Грина для адатомов 0 (подрешетка $A$ ) и 3 (подрешетка $B$

$$
\begin{aligned}
& D_{00}^{\sigma}(\omega)=\frac{1}{2}\left(1-C_{\sigma}\right) g_{+}^{\sigma}(\omega)+\frac{1}{2}\left(1+C_{\sigma}\right) g_{-}^{\sigma}(\omega), \\
& D_{33}^{\sigma}(\omega)=\frac{1}{2}\left(1+C_{\sigma}\right) g_{+}^{\sigma}+\frac{1}{2}\left(1-C_{\sigma}\right) g_{-}^{\sigma}(\omega),
\end{aligned}
$$


где

$$
g_{ \pm}^{\sigma}(\omega)=\left(\omega-\varepsilon_{ \pm \sigma}+i \Gamma_{m}\right)^{-1}
$$

и $\varepsilon_{ \pm \sigma}=\varepsilon_{\sigma} \pm t_{\sigma}, t_{\sigma}=\sqrt{\Delta_{\sigma}^{2}+z t^{2}}, C_{\sigma}=\left|\Delta_{\sigma}\right| / t_{\sigma}$. После соответствующих преобразований, аналогично [12] получим

$$
\begin{gathered}
\pi(a+b)=\operatorname{arccot}\left(\left(\varepsilon_{+\uparrow}-\varepsilon_{\mathrm{F}}\right) / \Gamma_{m}\right)+\operatorname{arccot}\left(\left(\varepsilon_{-\uparrow}-\varepsilon_{\mathrm{F}}\right) / \Gamma_{m}\right), \\
\pi(a-b)=\operatorname{arccot}\left(\left(\varepsilon_{+\downarrow}-\varepsilon_{\mathrm{F}}\right) / \Gamma_{m}\right)+\operatorname{arccot}\left(\left(\varepsilon_{-\downarrow}-\varepsilon_{\mathrm{F}} / \Gamma_{m}\right),\right. \\
-\pi(c-d)=C_{\downarrow}\left[\operatorname{arccot}\left(\left(\varepsilon_{+\downarrow}-\varepsilon_{\mathrm{F}}\right) / \Gamma_{m}\right)\right. \\
-\operatorname{arccot}\left(\left(\varepsilon_{-\downarrow}-\varepsilon_{\mathrm{F}} / \Gamma_{m}\right)\right], \\
-\pi(c+d)=C_{\uparrow}\left[\operatorname{arccot}\left(\left(\varepsilon_{+\uparrow}-\varepsilon_{\mathrm{F}}\right) / \Gamma_{m}\right)\right. \\
-\operatorname{arccot}\left(\left(\varepsilon_{-\uparrow}-\varepsilon_{\mathrm{F}}\right) / \Gamma_{m}\right] . \quad(\Pi 10)
\end{gathered}
$$

4. Для допированного ЭГ при $t=0$ и $\varepsilon^{*}-\varepsilon_{\mathrm{F}}=\delta$ имеем $\varepsilon_{A \uparrow, B \uparrow}-\varepsilon_{\mathrm{F}}=\delta \pm U(c-d) / 2-c z G$ и $\varepsilon_{A \downarrow, B \downarrow}-\varepsilon_{\mathrm{F}}=$ $=\delta \pm U(c+d) / 2-c z G$. Тогда при $c=0, d \neq 0($ ВСП) получаем

$$
\begin{aligned}
& E^{\prime}(0, d)=E_{0}-\frac{d^{2} U}{2} \\
& \quad+\frac{\Gamma_{m}}{\pi} \ln \frac{\left[(\delta+d U / 2)^{2}+\Gamma_{m}^{2}\right]\left[(\delta-d U / 2)^{2}+\Gamma_{m}^{2}\right]}{W^{4}},
\end{aligned}
$$

при $c \neq 0, d=0$ (ВЗП) имеем

$$
\begin{aligned}
& \left.E^{\prime}(c, 0)=E_{0}+\frac{c^{2}(U-z G)}{2}\right]+\frac{\Gamma_{m}}{\pi} \\
& \times \ln \frac{\left[(\delta+c(U / 2-z G))^{2}+\Gamma_{m}^{2}\right]\left[(\delta-c(U / 2-z G))^{2}+\Gamma_{m}^{2}\right]}{W^{4}},
\end{aligned}
$$

при $c=d=0$ (ПМ) получаем

$$
E^{\prime}(0,0)=E_{0}+\frac{2 \Gamma_{m}}{\pi} \ln \frac{\delta^{2}+\Gamma_{m}^{2}}{W^{2}} .
$$

Из уравнения $\bar{E}(0, d)=\bar{E}(c, 0)$ при $c=d$ находим условие $U=z G$. Выражения (П11) и (П12) при $d=0$ и $c=0$ соответственно переходят в уравнение (П13). Интересно отметить, что значения энергий (П11)-(П13) от знака параметра $\delta$ не зависят.

5. Как и ранее, будем считать плотность состояний металлической подложки $\rho_{\text {sub }}(\omega)=\rho_{m}=$ const. Тогда по аналогии с [17] для плотности состояний ЭГ (на элементарную ячейку) в рамках низкоэнергетической аппроксимации электронного спектра получим следующее выражение

$$
\begin{aligned}
\rho_{A B}^{\sigma}\left(\Omega_{\sigma}\right) & =\frac{\Gamma_{m}}{\pi \xi^{2}} \ln \frac{\left|\xi^{4}+B_{\sigma} \xi^{2}+C_{\sigma}\right|}{C_{\sigma}} \\
& +\frac{2 \Omega_{\sigma}}{\pi \xi^{2}}\left(\arctan \frac{2 \xi^{2}+B_{\sigma}}{4 \Gamma_{m} \Omega_{\sigma}}-\arctan \frac{B_{\sigma}}{4 \Gamma_{m} \Omega_{\sigma}}\right) .
\end{aligned}
$$

Здесь $\quad B_{\sigma}=-2\left(\Omega_{\sigma}^{2}-\Delta_{\sigma}^{2}-\Gamma_{m}^{2}\right), \quad C_{\sigma}=\left(\Omega_{\sigma}^{2}-\Delta_{\sigma}^{2}\right)^{2}$ $+\Gamma_{m}^{2}\left(\Gamma_{m}^{2}+2 \Delta_{\sigma}^{2}+2 \Omega_{\sigma}^{2}\right)$, остальные обозначения те же, что в формуле (20). При $a=1$ и $b=0$ имеем $\varepsilon_{\sigma}=0 \quad\left(\Omega_{\sigma}=\omega\right)$ и $\Delta_{\uparrow, \downarrow}=U(c \mp d) / 2-c z G$. Отсюда при $c=d \neq 0$ и $U=z G$ получаем $\Delta_{\sigma}^{2}(d U)^{2}$, так что $\rho_{A B}^{\uparrow}(\omega)=\rho_{A B}^{\downarrow}(\omega) \equiv \rho_{A B}(\omega)$ и уравнение $U=z G$ определяет границу ВСП- и ВЗП-состояний.

\section{Список литературы}

[1] A.H. Castro Neto, F. Guinea, N.M.R. Peres, K.S. Novoselov, A.K. Geim. Rev. Mod. Phys. 81, 109 (2009).

[2] V.N. Kotov, B. Uchoa, V.M. Pereira, A.H. Castro Neto, F. Guinea. Rev. Mod. Phys. 84, 1067 (2012).

[3] N. Swain, P. Majumdar. arXive: 1610.00695.

[4] M.V. Ulybyshev, P.V. Buividovich, M.I. Katsnelson, M.I. Polikarpov. Phys. Rev. Lett. 111, 056801 (2013).

[5] L. Wang, P. Corboz, M. Troyer. New J. Phys. 16, 103008 (2014).

[6] M. Hohenadler, F.P. Toldin, I.F. Herbut, F.F. Assaad. Phys. Rev. B 90, 085146 (2014).

[7] W. Wu, A.-M.S. Tremblay. Phys. Rev. B 89, 205128 (2014).

[8] L. Classen, I.F. Herbut, L. Janssen, M.M. Scherer. Phys. Rev. B 92, 035429 (2015).

[9] С.Ю. Давыдов. ФТТ 20, 1752 (1978).

[10] С.Ю. Давыдов. ФТП 48, 49 (2014).

[11] С.Ю. Давыдов. Теория адсорбции: метод модельных гамильтонианов. Изд-во СПбГЭТУ „ЛЭТИ“, СПб. (2013). $235 \mathrm{c}$.

[12] С.Ю. Давыдов. ФТТ 21, 2283 (1979).

[13] Д.И. Хомский. ФММ 29, 31 (1970).

[14] Е.В. Кузьмин, Г.А. Петраковский, Э.А. Завадский. Физика магнитоупорядоченного вещества. Наука, Новосибирск (1976). $283 \mathrm{c}$.

[15] Т. Мория. Спиновые флуктуации в магнетиках с коллективизированными электронами. Мир, М. (1988). 288 с.

[16] С.Ю. Давыдов. ФТП 46, 204 (2012).

[17] С.Ю. Давыдов. ФТТ 58, 779 (2016).

[18] J.E. Hirsh. Phys. Rev. Lett. B 53, 2327 (1984).

[19] V. Zhang, J. Callaway. Phys. Rev. B 39, 9397 (1989).

[20] J. van den Brink, M.B.J. Meinders, J. Lorenzana, R. Eder, G.A. Sawatzky. Phys. Rev. Lett. 75, 4658 (1995).

[21] P. Sengupta, A.W. Sandvik, D.K. Campbell. Phys. Rev. B 65, 155113 (2002).

[22] T.O. Wehling, E. Şaşığlu, C. Friedrich, A.I. Lichtenstein, M.I. Katsnelson, S. Blügel. Phys. Rev. Lett. 106, 236805 (2011).

[23] Ч. Киттель. Квантовая теория твердых тел. Наука, М. (1967). $492 \mathrm{c}$.

[24] С.Ю. Давыдов. ФТП 47, 97 (2013).

[25] Z.Y. Meng, T.C. Lang, S. Wessel, F.F. Assaad, A. Muramatsu. Nature 464, 847 (2010).

[26] S. Sorella, Y. Otsuka, S. Yunoki. Sci. Rep. 2, 992 (2012).

[27] A.G. Grushin, E.V. Castro, A. Cortijo, F. de Juan, M.A.H. Vozmediano, B. Valenzuela. Phys. Rev. B 87, 085136 (2013).

[28] X.Y. Xu, S. Wesse, Z.Y. Meng. Phys. Rev. B 94, 116105 (2016). 
[29] У. Харрисон. Электронная структура и свойства твердых тел. Мир, М. (1983). Т. 2. 334 с.

[30] Физические величины. Справочник / Под ред. И.С. Григорьева, Е.3. Мейлихова. Энергоатомиздат, М. (1991). $1232 \mathrm{c}$.

[31] В.Ю. Ирхин, Ю.П. Ирхин. Электронная структура, физические свойства и корреляционные эффекты в $d$ - и $f$-металлах и их соединениях. Екатеринбург, УрО РАН (2004). $472 \mathrm{c}$.

[32] R. Hovdena, A.W. Tsen, P. Liua, B.H. Savitzky, I.El Baggari, Y. Liu, W. Lu, Y. Sun, P. Kim, A.N. Pasupathy, L.F. Kourkoutis. Proc. Nat. Acad. Sci. USA 113, 11420 (2016).

[33] J.W.F. Venderbos, M. Manzardo, D.V. Efremov, J. van den Brink, C. Ortix. Phys. Rev. B 93, 045428 (2016).

[34] С.Ю. Давыдов. ЖТФ 86, 7, 145 (2016). 